\title{
Caracterização agronômica de mutantes de bananeira obtidos por meio da radiação gama
}

\author{
Edson Perito Amorim ( $\left.{ }^{*}\right)$; Rosa Karla Nogueira Pestana ( $\left.{ }^{2}\right)$; Sebastião de Oliveira e Silva $\left({ }^{2}\right)$; \\ Augusto Tulmann Neto ( $\left.{ }^{3}\right)$
}

(1) Embrapa Mandioca e Fruticultura, Rua Embrapa s/n, 44380-000 Cruz das Almas (BA), Brasil.

(2) Universidade Federal do Recôncavo da Bahia (UFRB), 44380-000 Cruz das Almas (BA), Brasil.

(3) Centro de Energia Nuclear na Agricultura (CENA/USP), Avenida Centenário 303, 13416-000 Piracicaba (SP), Brasil.

(*) Autor correspondente: edson@cnpmf.embrapa.br

Recebido: 25/maio/2011; Aceito: 16/dez./2011

\begin{abstract}
Resumo
A bananeira é uma das fruteiras de maior importância econômica, explorada quase exclusivamente por pequenos produtores. Constitui-se em fonte contínua de alimento e renda. Embora o Brasil figure como um dos maiores produtores de banana, a bananicultura nacional enfrenta sérios problemas nas fases de produção e pós-colheita, que limitam sua inserção no mercado internacional. Um dos fatores que levam a grandes perdas na produção é o tombamento resultante da altura elevada da planta das principais cultivares comerciais. Uma estratégia para a solução deste problema é a redução do porte por indução de mutação. O objetivo deste trabalho foi caracterizar mutantes de banana tipo Prata (cv's Pacovan e Preciosa) irradiadas, durante dois ciclos de produção, visando à seleção de plantas com porte baixo e boas características agronômicas. Plantas in vitro das duas cultivares foram irradiadas com raios gamas nas doses de 20 Gy ('Pacovan' - 200 mudas) e 30 Gy ('Preciosa' - 200 mudas), subcultivadas por quatro vezes e posteriormente avaliadas em campo durante dois ciclos de produção. Foram selecionados quatro mutantes de cada cultivar com altura inferior à média de altura das testemunhas, após dois ciclos de avaliação. Observou-se que em alguns desses mutantes houve maior precocidade e maior massa do cacho quando comparados com as testemunhas. Pelos resultados, é possível selecionar plantas mutantes com características agronômicas superiores, tanto para a 'Pacovan' quanto para a 'Preciosa', submetidas à radiação gama.
\end{abstract}

Palavras-chave: Musa spp., mutagênese in vitro, seleção de mutantes.

\section{Agronomic characterization of banana mutants obtained by gamma radiation}

\section{Abstract}

Banana is one of the most economically important fruit, explored almost exclusively by small producers as a continuous source of food and income. Although Brazil is one of the main banana producers, the national banana production is undergoing serious problems especially in the phases of production and post-harvest limiting its participation in the international market. One of the main factors leading to great production losses is the toppling over due to the tall height of plants of main commercial cultivars. A strategy to solve this problem is reducing height by inducing mutation. The objective of the present work was to characterize irradiated Prata type banana mutants (cvs. Pacovan and Preciosa) during two production cycles in order to select short plants in height with good agronomic characteristics. In vitro plants of both cultivars were irradiated with gamma rays in the doses of 20 Gy ('Pacovan', 200 plants) and 30 Gy ('Preciosa', 200 plants) subcultivated four times and afterwards evaluated in the field during two production cycles. Four possible mutants were selected from each cultivar with height smaller than the average height of the controls after two evaluation cycles. It was observed that some of these mutants presented greater precocity and bunch weight compared to the controls. From the results obtained it is possible to select mutant plants with superior agronomic characteristics for 'Pacovan' as well as 'Preciosa' submitted to gamma radiation.

Key words: Musa spp., in vitro mutagenesis, selection of mutants. 


\section{INTRODUÇÃO}

A bananeira é uma das fruteiras de maior importância econômica, explorada quase exclusivamente por pequenos produtores. Constitui-se em uma fonte contínua de alimento e renda, além de proporcionar a fixação da mão de obra no meio rural (SiLva et al., 2003). O Brasil se destaca como o quinto produtor mundial de banana, com produção aproximada de 7,0 milhôes de toneladas em 2009, em área cultivada próxima a 512 mil hectares (FAO, 2011).

Embora o Brasil figure como um dos maiores produtores, a bananicultura nacional enfrenta sérios problemas na produção e pós-colheita, que limitam os ganhos. Um dos fatores que levam a grandes perdas é o tombamento, provocado pela altura elevada da planta das principais cultivares comerciais.

Uma estratégia para a solução deste problema é o desenvolvimento de novas cultivares mediante o melhoramento genético, a partir de hibridação e seleção na progênie (Silva et al., 2002; Donato et al., 2006). No entanto, no caso específico da bananeira, existem alguns obstáculos no melhoramento genético, entre eles, a elevada esterilidade feminina e o reduzido número de sementes produzidas.

Em situaçôes como esta, e no caso onde existem limitaçóes na variabilidade genética natural disponível, ou dificuldades relacionadas às aplicaçôes de métodos tradicionais de melhoramento, uma ferramenta que pode superar estas barreiras é a indução de mutação (Pestana et al., 2010).

Dentre as características mutantes que se observam em Musa spp., quando se utiliza a induçáo de mutação, uma das mais frequentes refere-se à redução da altura de planta (TANg e TAI, 2001).

Em bananeira, alguns trabalhos têm sido realizados visando à geração de variabilidade genética para o melhoramento, a partir de irradiação com agentes físicos e ou químicos (Ho et al., 2001; Pestana et al., 2010). No entanto, apenas duas cultivares desenvolvidas por meio de raios gama foram registradas na Agência Internacional de Energia Atômica, a primeira a 'Klue Hom Thong KU1' que apresenta elevada massa do cacho e a 'Novaria' com ciclo precoce e qualidade dos frutos (FAO, 2011).

Este trabalho teve como objetivo caracterizar mutantes de banana tipo Prata (cv's 'Pacovan' e 'Preciosa') durante dois ciclos de produção, visando à seleção de plantas com porte baixo e boas características agronômicas.

\section{MATERIAL E MÉTODOS}

\section{Material Genético}

Plantas in vitro de bananeira das cultivares de porte alto 'Pacovan' (AAB) e 'Preciosa' (AAAB), com aproximadamente $5 \mathrm{~cm}$ de comprimento e com 4-5 primórdios foliares foram utilizadas para indução de mutação visando à seleção de plantas baixas.

\section{Irradiação gama de gemas in vitro}

Aproximadamente 200 gemas in vitro de cada cultivar foram irradiadas utilizando fonte $\mathrm{Co}^{60}$ no Centro de Energia Nuclear na Agricultura (CENA, USP). As doses utilizadas foram 20 Gy para a cultivar Pacovan e 30 Gy para Preciosa, com taxas de 1,322 kgy h${ }^{-1}$. Para cada cultivar, dez gemas foram usadas como testemunhas, as quais também foram enviadas ao CENA/USP, porém sem exposição ao $\mathrm{Co}^{60}$.

As gemas irradiadas foram transferidas para meio de cultura básico MS, solidificado com 2,2 $\mathrm{g} \mathrm{L}^{-1}$ de Phytagel, e suplementado com $30 \mathrm{~g}$ de sacarose e benzilaminopurina (BAP) na concentração de $3,0 \mathrm{mg} \mathrm{L}^{-1} \mathrm{e} \mathrm{pH} \mathrm{5,8.} \mathrm{As}$ culturas foram mantidas em sala de crescimento com temperatura controlada de $27 \pm 2{ }^{\circ} \mathrm{C}$ e fotoperíodo de 16 horas de luz. As mudas foram submetidas a quatro subcultivos, realizados em intervalos de 30 dias.

Após os subcultivos, as plantas foram enraizadas em meio MS, com adição de $0,25 \mathrm{mg} \mathrm{L}^{-1}$ de ácido naftaleno acético (ANA) e $8 \mathrm{~g} \mathrm{~L}^{-1}$ de ágar como geleificante, com o $\mathrm{pH}$ ajustado para 5,8 e levadas para a sala de crescimento com fotoperíodo de 16 horas, intensidade luminosa de 40 $\mu \mathrm{mol} \mathrm{m} \mathrm{s}^{-1}$ e temperatura de $26 \pm 2{ }^{\circ} \mathrm{C}$, onde permaneceram por um período de 35-40 dias. As plantas já enraizadas foram levadas para telado, onde foram aclimatadas em tubetes contendo substrato PlantMax e em telado com sombrite 50\%, com controle de luminosidade e irrigação.

Uma pré-seleção foi realizada em telado, utilizando-se como critério a identificaçáa de plantas com altura inferior às testemunhas em, no mínimo, $10 \%$. Foram selecionadas 190 plantas entre as 3.200 irradiadas da 'Preciosa' e entre as 1.200 plantas irradiadas da 'Pacovan' selecionaram-se 179. Estas plantas, juntamente com 53 outras testemunhas (36 de 'Pacovan' e 17 de 'Preciosa'), foram caracterizadas agronomicamente.

\section{Caracterização agronômica dos mutantes}

As plantas selecionadas em telado foram plantadas em períodos distintos no campo experimental da Embrapa Mandioca e Fruticultura, e as diferentes épocas de instalação do experimento ficaram condicionadas ao vigor das mudas. A implantação do experimento foi realizada sem adoção de delineamento experimental, e o objetivo principal do trabalho foi selecionar $10 \%$ das melhores plantas em um grupo relativamente grande de bananeiras.

Nas duas áreas experimentais, o plantio foi feito no espaçamento $3 \mathrm{~m} \mathrm{x} 4 \mathrm{~m}$, e a adubação de fundação e cobertura foi realizada de acordo com as recomendaçôes técnicas. 
As avaliações foram realizadas no período do florescimento, avaliando-se 369 plantas irradiadas e 53 plantas não irradiadas (testemunhas) em dois ciclos de produçáo, quanto às seguintes características agronômicas: número de dias do plantio ao florescimento, do plantio à colheita e número de dias do florescimento à colheita; diâmetro do pseudocaule $(\mathrm{cm})$; altura da planta $(\mathrm{m})$; número de folhas vivas na floração e de filhos; massa do cacho $(\mathrm{kg})$, das pencas $(\mathrm{kg})$ e massa média dos frutos $(\mathrm{g})$; número de frutos por cacho; comprimento do fruto da segunda e da penúltima penca $(\mathrm{cm})$; diâmetro do fruto da segunda penca e da penúltima penca $(\mathrm{mm})$; número de pencas e de folhas vivas na colheita; comprimento do engaço $(\mathrm{cm})$; diâmetro de engaço $(\mathrm{mm})$; e presença de Sigatoka-amarela na floração e na colheita utilizando a escala proposta por STOVER (1972) modificada por GAuHL et al. (1993).

\section{Seleção dos mutantes para porte baixo}

Para selecionar $10 \%$ das plantas com menor porte inicialmente foi realizada uma classificaçáo individual das plantas da população, para as características consideradas importantes na seleção de genótipos de bananeira, como menor porte de planta, menor número de dias do plantio ao florescimento e maior massa do cacho. Os dados obtidos para essas características foram organizados em ordem crescente (para altura de planta e números de dias para emissão do cacho) e decrescente (para massa do cacho), obtendo-se o número da classificação de cada planta.

Em seguida, multiplicou-se o número da classificação de cada planta pela massa correspondente a cada característica. Para altura de planta foi dado peso seis por ser considerada a mais relevante e para número de dias do plantio ao florescimento e massa do cacho peso dois. No fim desse processo, obteve-se a pontuação final, para cada planta, resultado do uso da fórmula $y=[0,6 \mathrm{x}$ (classificação de altura) + 0,2 x (classificação da emissáo de cacho) + $0,2 \times$ (classificação da massa do cacho)]. Em cada ciclo, a pontuaçáo final foi ordenada crescentemente, obtendo-se a classificação final. As plantas selecionadas foram aquelas que estavam presentes entre as $10 \%$ melhores classificadas.

\section{Análise estatística dos dados}

Inicialmente, os dados foram submetidos à análise de variância considerando o esquema fatorial 2 × 2 × 2 , duas cultivares (Pacovan e Preciosa), dois tratamentos (testemunha e irradiado) e dois ciclos (1 e 2). Foi também realizada uma análise de variância considerando os mutantes selecionados. Para isso, considerou-se o delineamento inteiramente casualizado no esquema fatorial $2 \times 2$, dois tratamentos (mutantes selecionados e testemunhas) e dois ciclos de produção. Para as duas análises, as médias foram comparadas pelo teste $\mathrm{F}$ a 5\% de probabilidade. As análises foram realizadas utilizando-se o programa SAS (2002).

\section{RESULTADOS E DISCUSSÃO}

Houve diferenças significativas entre cultivares $(p<0,05)$ para quase todos os caracteres avaliados nos dois ciclos de produção, à exceção da altura de planta (ALP), número de filhos (NFI), número de frutos (NFR) e comprimento do fruto da segunda e penúltima pencas (CFS e CFP), número de pencas (NPE) e diâmetro do engaço (DEG) (Tabela 1). Comportamento semelhante também foi observado para a fonte de variação Tratamentos (cultivares submetidas ou não a irradiação), onde somente nas variáveis número de dias do plantio a colheita (NPC), número de dias do plantio ao florescimento (NFC), altura de planta (ALP), número de folhas e de filhos (NFO e NFI), comprimento do fruto da penúltima penca (CFP), comprimento do engaço (CEG) e Sigatoka-amarela na colheita (SIC) não houve diferenças estatísticas.

$\mathrm{Na}$ fonte de variação Ciclo todas as variáveis foram significativas $(\mathrm{p}<0,01)$, exceto o número de dias do florescimento a colheita (NFC), comprimento do fruto da penúltima penca (CFP), diâmetro do fruto da segunda penca (DFS) e Sigatoka-amarela na floração (SIG) (Tabela 1).

$\mathrm{Na}$ interaçáo cultivares $\mathrm{x}$ tratamentos (com e sem irradiação) houve diferença estatística somente para diâmetro do fruto da segunda penca (DFS), número de folhas vivas na colheita (NFV), comprimento do engaço (CEG), e Sigatoka-amarela na floração (SIG). Da mesma forma, na interação cultivares $\mathrm{x}$ ciclos, poucas variáveis foram significativas, entre eles o número de folhas na floração (NFO), número de folhas vivas na colheita (NFV), comprimento do engaço (CEG), diâmetro do engaço (DEG) e Sigatoka-amarela na colheita (SIC) (Tabela 1).

Por meio da seleção realizada utilizando-se o índice de peso segundo a importância das características altura de planta, número de dias do plantio ao florescimento e massa do cacho, foi possível selecionar oito prováveis mutantes, sendo quatro de Preciosa e quatro de Pacovan.

Visando confirmar que estes prováveis mutantes diferem estatisticamente das respectivas testemunhas não irradiadas para as características agronômicas mensuradas, foi realizada uma nova análise de variância, agora considerando o efeito de tratamentos com e sem irradiaçáo e ciclo.

Pelos resultados percebe-se que a fonte de variação Tratamentos foi significativa apenas para diâmetro do engaço (DIA); altura de planta (ALP); número de frutos (NFR); diâmetro do engaço (DEG) e Sigatoka-amarela na floração (SIG). Desta forma, é possível inferir que as plantas selecionadas diferem das testemunhas para estes caracteres (Tabela 2). 
Tabela 1. Análise de variância para variáveis de crescimento e produção em dois ciclos nas cultivares Pacovan e Preciosa irradiadas e não irradiadas

\begin{tabular}{|c|c|c|c|c|c|c|c|c|}
\hline \multirow{2}{*}{ FV } & \multirow{2}{*}{ GL } & \multicolumn{7}{|c|}{ QM } \\
\hline & & NPF & NPC & NFC & DIA & ALP & NFO & NFI \\
\hline Cultivares (Cv) & 1 & $114,3^{* *}$ & $139,6^{* *}$ & $7,4^{* *}$ & $148,1^{* *}$ & $0,1^{\text {ns }}$ & $1,5^{* *}$ & $0,2^{\text {ns }}$ \\
\hline Tratamentos $(\mathrm{T})$ & 1 & $12,8^{*}$ & $8,1^{\text {ns }}$ & $2,6^{\mathrm{ns}}$ & $52,6^{* *}$ & $0,01^{\mathrm{ns}}$ & $0,3^{\text {ns }}$ & $0,01^{\text {ns }}$ \\
\hline Ciclos (C) & 1 & $4585,7^{* *}$ & $3586,1^{* *}$ & $0,9^{\text {ns }}$ & $100,7^{* *}$ & $38,1^{* *}$ & $23,8^{* *}$ & $19,5^{* *}$ \\
\hline $\mathrm{Cv} \times \mathrm{T}$ & 1 & $1,8^{\text {ns }}$ & $2,7^{\text {ns }}$ & $0,00^{\text {ns }}$ & $0,4^{\text {ns }}$ & $0,04^{\mathrm{ns}}$ & $0,2^{\text {ns }}$ & $0,1^{\text {ns }}$ \\
\hline $\mathrm{Cv} \times \mathrm{C}$ & 1 & $0,1^{\text {ns }}$ & $0,2^{\text {ns }}$ & $0,07^{\text {ns }}$ & $5,7^{\mathrm{ns}}$ & $0,04^{\mathrm{ns}}$ & $1,3^{* *}$ & $0,1^{\text {ns }}$ \\
\hline $\mathrm{T} \times \mathrm{C}$ & 1 & $2,8^{\text {ns }}$ & $4,7^{\text {ns }}$ & $0,4^{\mathrm{ns}}$ & $0,6^{\mathrm{ns}}$ & $0,2^{\text {ns }}$ & $0,3^{\text {ns }}$ & $0,2^{\text {ns }}$ \\
\hline $\mathrm{Cv} \times \mathrm{T} \times \mathrm{C}$ & 1 & $0,2^{\text {ns }}$ & $0,6^{\text {ns }}$ & $0,01^{\text {ns }}$ & $15,3^{\text {ns }}$ & $0,1^{\text {ns }}$ & $1,8^{* *}$ & $0,1^{\text {ns }}$ \\
\hline Erro & 836 & 2,7 & 2,5 & $0,10^{\text {ns }}$ & 5,6 & 0,2 & 0,1 & 0,1 \\
\hline CV (\%) & & 7,4 & 6,4 & 8,6 & 12,9 & 13,0 & 11,8 & 21,1 \\
\hline Média & & 497,5 & 628,8 & 133,8 & 18,3 & 3,1 & 9,8 & 2,6 \\
\hline \multirow{2}{*}{ FV } & \multirow{2}{*}{ GL } & \multicolumn{7}{|c|}{ QM } \\
\hline & & MCA & MPE & NFR & MMF & CFS & CFP & DFS \\
\hline Cultivares (Cv) & 1 & $75,3^{* *}$ & $83,5^{* *}$ & $0,2^{\mathrm{ns}}$ & $15468,3^{* *}$ & $25,3^{\mathrm{ns}}$ & $23,7^{\mathrm{ns}}$ & $300,5^{* *}$ \\
\hline Tratamentos $(\mathrm{T})$ & 1 & $210,8^{* *}$ & $170,9^{* *}$ & $17,5^{* *}$ & $13785,2^{* *}$ & $66,9^{* *}$ & $40,6^{\mathrm{ns}}$ & $278,0^{* *}$ \\
\hline Ciclos (C) & 1 & $144,5^{* *}$ & $145,2^{* *}$ & $11,2^{* *}$ & $12361,4^{* *}$ & $39,6^{*}$ & $6,2^{\text {ns }}$ & $84,8^{\text {ns }}$ \\
\hline $\mathrm{Cv} \times \mathrm{T}$ & 1 & $15,5^{\text {ns }}$ & $18,3^{\text {ns }}$ & $0,2^{\text {ns }}$ & $4128,7^{\mathrm{ns}}$ & $4,2^{\text {ns }}$ & $17,1^{\mathrm{ns}}$ & $107,3^{*}$ \\
\hline $\mathrm{Cv} \times \mathrm{C}$ & 1 & $0,1^{\text {ns }}$ & $0,2^{\text {ns }}$ & $1,1^{\text {ns }}$ & $1017,7^{\mathrm{ns}}$ & $10,6^{\text {ns }}$ & $15,7^{\mathrm{ns}}$ & $1,2^{\text {ns }}$ \\
\hline$T \times C$ & 1 & $1,6^{\text {ns }}$ & $0,7^{\text {ns }}$ & $0,1^{\text {ns }}$ & $1271,8^{\text {ns }}$ & $0,00^{\text {ns }}$ & $3,0^{\text {ns }}$ & $14,4^{\text {ns }}$ \\
\hline $\mathrm{Cv} \times \mathrm{T} \times \mathrm{C}$ & 1 & $11,2^{\text {ns }}$ & $9,3^{\text {ns }}$ & $0,4^{\mathrm{ns}}$ & $1045,2^{\text {ns }}$ & $1,5^{\mathrm{ns}}$ & $5,7^{\mathrm{ns}}$ & $6,4^{\mathrm{ns}}$ \\
\hline Erro & 836 & 8,1 & 7,1 & 1,0 & 1327,8 & 6,7 & 20,1 & 22,7 \\
\hline CV (\%) & & 42,8 & 45,3 & 12,8 & 38,6 & 19,0 & 35,2 & 14,4 \\
\hline Média & & 6,7 & 5,9 & 61,7 & 94,3 & 13,6 & 12,7 & 33,1 \\
\hline \multirow{2}{*}{ FV } & \multirow{2}{*}{ GL } & \multicolumn{7}{|c|}{ QM } \\
\hline & & DFP & $\mathrm{NPE}^{2}$ & $\mathrm{NFV}^{2}$ & CEG & DEG & $\mathrm{SIG}^{3}$ & $\mathrm{SIC}^{3}$ \\
\hline Cultivares (Cv) & 1 & $261,2^{* *}$ & $0,1^{\text {ns }}$ & $5,5^{* *}$ & $466,4^{*}$ & $173,3^{\text {ns }}$ & $4,0^{* *}$ & $3,6^{* *}$ \\
\hline Trat $(\mathrm{T})$ & 1 & $188,4^{* *}$ & $0,4^{* *}$ & $3,2^{*}$ & $212,1^{\text {ns }}$ & $290,2^{*}$ & $0,5^{* *}$ & $0,1^{\mathrm{ns}}$ \\
\hline Ciclos (C) & 1 & $179,2^{*}$ & $0,4^{* *}$ & $11,2^{* *}$ & $3867,2^{* *}$ & $1508,1^{* *}$ & $0,15^{\mathrm{ns}}$ & $4,2^{* *}$ \\
\hline Cv $x T$ & 1 & $77,8^{\text {ns }}$ & $0,03^{\mathrm{ns}}$ & $2,9^{*}$ & $345,4^{*}$ & $2,2^{\text {ns }}$ & $0,6^{* *}$ & $0,1^{\mathrm{ns}}$ \\
\hline $\mathrm{Cv} \times \mathrm{C}$ & 1 & $33,1^{\text {ns }}$ & $0,05^{\mathrm{ns}}$ & $4,0^{*}$ & $371,5^{*}$ & $542,4^{* *}$ & $0,04^{\text {ns }}$ & $2,0^{* *}$ \\
\hline$T \times C$ & 1 & $10,3^{\text {ns }}$ & $0,00^{\text {ns }}$ & $0,9^{\text {ns }}$ & $54,7^{\text {ns }}$ & $0,5^{\text {ns }}$ & $0,04^{\mathrm{ns}}$ & $0,2^{\text {ns }}$ \\
\hline $\mathrm{Cv} \times \mathrm{T} \times \mathrm{C}$ & 1 & $73,5^{\text {ns }}$ & $0,01^{\mathrm{ns}}$ & $0,1^{\mathrm{ns}}$ & $23,2^{\text {ns }}$ & $57,3^{\text {ns }}$ & $0,01^{\mathrm{ns}}$ & $0,2^{\mathrm{ns}}$ \\
\hline Erro & 836 & 27,0 & 0,04 & 0,6 & 85,4 & 54,4 & 0,07 & 0,2 \\
\hline CV (\%) & & 16,2 & 8,8 & 52,2 & 27,1 & 16,1 & 29,9 & 44,8 \\
\hline Média & & 32,1 & 5,3 & 2,9 & 34,1 & 45,8 & 0,3 & 0,7 \\
\hline
\end{tabular}

NPF: número de dias do plantio à floraçáo; NPC: número de dias do plantio à colheita; NFC: número de dias da floraçâo à colheita; DIA: diâmetro do pseudocaule (cm); ALP: altura de planta (m); NFO: número de folhas na floraçáo; NFI: número de filhos; MCA: massa de cacho (kg); MPE: massa de pencas (kg); NFR: número de frutos; MMF: massa média do fruto (g); CFS: comprimento do fruto da segunda penca (cm); CFP: comprimento do fruto da penúltima penca (cm); DFS: diâmetro do fruto da segunda penca $(\mathrm{mm})$; DFP: diâmetro do fruto da penúltima penca $(\mathrm{mm})$; NPE: número de pencas; NFV: número de folhas vivas na colheita; CEG: comprimento do engaço $(\mathrm{cm})$; DEG: diâmetro do engaço $(\mathrm{cm})$; SIG: presença de Sigatoka-amarela na floração; SIC: presença de Sigatoka-amarela na colheita. ns: Não significativo. * Significativo a 5\% de probabilidade. ${ }^{* *}$ Significativo a $1 \%$ de probabilidade. $\left({ }^{2}\right)$ transformado para raiz $(\mathrm{x}) .\left(^{3}\right)$ transformado para raiz $(\mathrm{x}+0,5)$.

A fonte de variação Ciclo foi significativa para uma série de variáveis, com destaque para número de dias do plantio a floração e do plantio a colheita (NFC e NPC); altura de planta (ALP) e massa do cacho (MCA) (Tabela 2).

Foi observada significância para a fonte de variaçáo Tratamentos x Ciclos, para as características massa do cacho e da penca (MCA e MPE) e massa média dos frutos (MMF), indicando que há comportamento diferenciado das cultivares em funçáo do ciclo de produçáo. A MCA, MPE e a MMF foram maiores nas plantas selecionadas que nas testemunhas não irradiadas, no primeiro ciclo. Por outro lado, no segundo ciclo, a situação se inverteu para a MCA e MPE, mantendo-se semelhante apenas para a MMF (Tabela 3).

Para a cultivar Preciosa, percebe-se que a fonte de variaçáo Tratamentos foi significativa apenas para número de dias do plantio a floraçáo e do plantio a colheita (NFC e NPC); altura de planta (ALP) e número de frutos (NFR) (Tabela 4). Desta forma, é possível inferir que as plantas selecionadas diferem das testemunhas para estes caracteres. A fonte de variação Ciclos foi significativa para as variáveis número de dias do plantio à floração e do plantio à colheita (NFC e NPC); altura de planta (ALP); número de folhas vivas e filhos na floração (NFO e NFI) e diâmetro do engaço (DEG). 
Tabela 2. Análise de variância para variáveis de crescimento e produção em dois ciclos para as plantas irradiadas selecionadas e testemunhas na cultivar Pacovan

\begin{tabular}{|c|c|c|c|c|c|c|c|c|}
\hline \multirow{2}{*}{ FV } & \multirow{2}{*}{ GL } & \multicolumn{7}{|c|}{ QM } \\
\hline & & NPF & NPC & NF & DIA & ALP & NFO & NFI \\
\hline Tratamentos $(\mathrm{T})$ & 1 & $7,97^{\mathrm{ns}}$ & $4,79^{\text {ns }}$ & $0,11^{\mathrm{ns}}$ & $42,53^{* *}$ & $2,13^{* *}$ & $0,07^{\text {ns }}$ & $0,12^{\text {ns }}$ \\
\hline Ciclos (C) & 1 & $344,63^{* *}$ & $274,33^{* *}$ & $0,06^{\mathrm{ns}}$ & $30,83^{* *}$ & $2,63^{* *}$ & $0,95^{* *}$ & $1,40^{* *}$ \\
\hline $\mathrm{T} \times \mathrm{C}$ & 1 & $3,82^{\text {ns }}$ & $4,68^{\text {ns }}$ & $0,07^{\text {ns }}$ & $0,23^{\text {ns }}$ & $0,07^{\text {ns }}$ & $0,01^{\mathrm{ns}}$ & $0,08^{\text {ns }}$ \\
\hline Erro & 76 & 2,73 & 3,88 & 1,30 & 4,34 & 0,08 & 0,07 & 0,10 \\
\hline CV (\%) & & 7,93 & 8,30 & 9,90 & 11,53 & 17,56 & 8,50 & 19,50 \\
\hline Média & & 450,88 & 578,55 & 133,9 & 18,06 & 3,06 & 9,49 & 2,81 \\
\hline \multirow{2}{*}{ FV } & \multirow{2}{*}{ GL } & \multicolumn{7}{|c|}{ QM } \\
\hline & & MCA & MPE & NFR & MMF & CFS & CFP & DFS \\
\hline Tratamentos $(\mathrm{T})$ & 1 & $2,98^{\mathrm{ns}}$ & $1,22^{\mathrm{ns}}$ & $5,10^{*}$ & $1201,95^{\mathrm{ns}}$ & $0,61^{\mathrm{ns}}$ & $0,50^{\text {ns }}$ & $23,11^{\mathrm{ns}}$ \\
\hline Ciclos (C) & 1 & $49,88^{* *}$ & $44,90^{* *}$ & $1,51^{\mathrm{ns}}$ & $8525,22^{* *}$ & $10,51^{\mathrm{ns}}$ & $27,61^{* *}$ & $39,67^{\text {ns }}$ \\
\hline$T \times C$ & 1 & $24,46^{*}$ & $19,67^{*}$ & $0,69^{\text {ns }}$ & $4913,12^{*}$ & $0,61^{\mathrm{ns}}$ & $4,51^{\mathrm{ns}}$ & $33,37^{\text {ns }}$ \\
\hline Erro & 76 & 4,55 & 3,65 & 0,77 & 797,08 & 5,12 & 3,41 & 15,48 \\
\hline CV (\%) & & 29,15 & 29,89 & 10,78 & 29,12 & 16,14 & 14,36 & 11,79 \\
\hline Média & & 7,32 & 6,40 & 67,35 & 96,94 & 14,01 & 12,86 & 33,39 \\
\hline \multirow{2}{*}{ FV } & \multirow{2}{*}{ GL } & \multicolumn{7}{|c|}{ QM } \\
\hline & & DFP & NPE & NFV & CEG & DEG & SIG & SIC \\
\hline Tratamentos $(\mathrm{T})$ & 1 & $36,45^{\text {ns }}$ & $0,06^{\text {ns }}$ & $0,05^{\text {ns }}$ & $53,90^{\text {ns }}$ & $299,02^{* *}$ & $0,63^{*}$ & $0,04^{\text {ns }}$ \\
\hline Ciclos (C) & 1 & $45,00^{\text {ns }}$ & $0,00^{\text {ns }}$ & $4,55^{* *}$ & $185,03^{\text {ns }}$ & $66,01^{\mathrm{ns}}$ & $0,02^{\text {ns }}$ & $1,45^{*}$ \\
\hline $\mathrm{T} \times \mathrm{C}$ & 1 & $36,45^{\text {ns }}$ & $0,00^{\text {ns }}$ & $0,13^{\text {ns }}$ & $20,33^{\text {ns }}$ & $35,56^{\text {ns }}$ & $0,16^{\text {ns }}$ & $0,01^{\text {ns }}$ \\
\hline Erro & 76 & 13,41 & 0,02 & 0,49 & 109,53 & 40,92 & 0,09 & 0,24 \\
\hline CV (\%) & & 11,32 & 6,66 & 42,92 & 28,03 & 13,49 & 33,94 & 42,63 \\
\hline Média & & 32,35 & 5,63 & 3,26 & 37,34 & 47,43 & 0,39 & 1,09 \\
\hline
\end{tabular}

NPF: número de dias do plantio a floração; NPC: número de dias do plantio a colheita; NFC: número de dias da floração a colheita; DIA: diâmetro do pseudocaule (cm); ALP: altura de planta (m); NFO: número de folhas na floração; NFI: número de filhos; MCA: peso de cacho (kg); MPE: massa de pencas (kg); NFR: número de frutos; MMF: massa média do fruto (g); CFS: comprimento do fruto da segunda penca (cm); CFP: comprimento do fruto da penúltima penca (cm); DFS: diâmetro do fruto da segunda penca $(\mathrm{mm})$; DFP: diâmetro do fruto da penúltima penca $(\mathrm{mm})$; NPE: número de pencas; NFV: número de folhas vivas na colheita; CEG: comprimento do engaço $(\mathrm{cm})$; DEG: diâmetro do engaço $(\mathrm{cm})$; SIG: presença de Sigatoka-amarela na floraçăo; SIC: presença de Sigatoka-amarela na colheita. ns: Năo significativo. * Significativo a 5\% de probabilidade. ${ }^{* *}$ Significativo a $1 \%$ de probabilidade. $\left({ }^{2}\right)$ transformado para raiz $(\mathrm{x}) .\left(^{3}\right)$ transformado para raiz $(\mathrm{x}+0,5)$.

Tabela 3. Análise de variância para o desdobramento da interação tratamentos x ciclo, para variáveis de produção da cultivar Pacovan, provenientes de plantas selecionadas e irradiadas, no primeiro e segundo ciclos de produção

\begin{tabular}{lcccc} 
& & \multicolumn{2}{c}{ Ciclo 2} \\
Variáveis & Selecionadas 1 & Testemunhas & Selecionadas & Testemunhas \\
MCA & $8,98 \mathrm{a}$ & $7,78 \mathrm{a}$ & $4,50 \mathrm{~b}$ & $6,99 \mathrm{a}$ \\
MPE & $8,10 \mathrm{a}$ & $6,86 \mathrm{a}$ & $3,95 \mathrm{~b}$ & $6,01 \mathrm{a}$ \\
MMF & $138,84 \mathrm{a}$ & $99,79 \mathrm{~b}$ & $78,31 \mathrm{a}$ & $91,51 \mathrm{a}$ \\
\hline
\end{tabular}

MCA: massa de cacho (kg) e de pencas (MPE); MMF: massa média do fruto (g). Médias seguidas da mesma da letra na horizontal, dentro de cada ciclo, nấo diferem estatisticamente entre si pelo teste de F a $5 \%$ de probabilidade.

Não foi observada significância para a fonte de variação Tratamentos x Ciclos, o que indica que não há comportamento diferenciado das plantas da cultivar Preciosa irradiada e não irradiada, em função do ciclo de produção (Tabela 4).

Especificamente para 'Pacovan' é possível observar que a altura de planta foi menor nas plantas selecionadas que nas testemunhas; assim como o que ocorreu para número de frutos (Tabela 5). Bermúdez et al. (2000), trabalhando com mutantes da cultivar FHIA-21, observaram no primeiro ciclo, frequência geral de variação de $4,8 \%$, e as características mais alteradas foram a altura de planta e o número de frutos por cacho.

Para número de dias do plantio à colheita e do plantio à floração, não foram observadas diferenças significativas entre plantas selecionadas e testemunha da cultivar Pacovan.

Para a cultivar Preciosa, foi possível observar que as plantas irradiadas foram mais precoces que as testemunhas em 56 dias, além de seu menor porte (Tabela 5). Donato et al. (2006) em Guanambi (BA) apontam médias de 237 dias e 412 dias (primeiro e segundo ciclos) para Preciosa não irradiada, valores inferiores aos apresentados neste estudo, tanto para o tratamento irradiado quanto para a testemunha.

Os mutantes selecionados estão em avaliação em diferentes regiōes edafoclimáticas brasileiras visando quantificar seu comportamento agronômico para futuro lançamento como material comercial para o agronegócio da banana, disponibilizando novas opçôes para os agricultores. 
Tabela 4. Análise de variância para variáveis de crescimento e produção da cultivar Preciosa, provenientes de plantas selecionadas e irradiadas, no primeiro e segundo ciclos de produção

\begin{tabular}{|c|c|c|c|c|c|c|c|c|}
\hline \multirow{2}{*}{ FV } & \multirow{2}{*}{ GL } & \multicolumn{7}{|c|}{ QM } \\
\hline & & NPF & NPC & NFC & DIA & ALP & NFO & NFI \\
\hline Trat (T) & 1 & $9,22^{*}$ & $9,79 * *$ & $0,00^{\text {ns }}$ & $12,39^{\text {ns }}$ & $1,10^{* *}$ & $0,24^{\mathrm{ns}}$ & $0,06^{\text {ns }}$ \\
\hline Ciclo (C) & 1 & $377,22^{* *}$ & $305,16^{* *}$ & $0,01^{\mathrm{ns}}$ & $10,74^{\text {ns }}$ & $2,09^{* *}$ & $3,09^{* *}$ & $0,81^{* *}$ \\
\hline $\mathrm{T} \times \mathrm{C}$ & 1 & $0,01^{\text {ns }}$ & $0,14^{\text {ns }}$ & $0,10^{\text {ns }}$ & $4,02^{\text {ns }}$ & $0,00^{\text {ns }}$ & $0,22^{\mathrm{ns}}$ & $0,19^{\text {ns }}$ \\
\hline Erro & 38 & 1,56 & 1,04 & 0,88 & 3,09 & 0,06 & 0,18 & 0,09 \\
\hline CV (\%) & & 5,66 & 4,06 & 7,95 & 9,04 & 8,04 & 13,13 & 19,64 \\
\hline Média & & 502,05 & 640,83 & 140,67 & 19,46 & 2,98 & 10,62 & 2,55 \\
\hline \multirow{2}{*}{ FV } & \multirow{2}{*}{ GL } & \multicolumn{7}{|c|}{ QM } \\
\hline & & PCA & PPE & NFR & MMF & CFS & CFP & DFS \\
\hline Trat (T) & 1 & $22,70^{\text {ns }}$ & $21,96^{\text {ns }}$ & $2,99^{*}$ & $843,78^{\mathrm{ns}}$ & $0,20^{\text {ns }}$ & $0,00^{\text {ns }}$ & $54,08^{\text {ns }}$ \\
\hline Ciclo (C) & 1 & $8,09^{\text {ns }}$ & $10,33^{\text {ns }}$ & $0,22^{\text {ns }}$ & $596,99^{\text {ns }}$ & $0,36^{\mathrm{ns}}$ & $1,57^{\mathrm{ns}}$ & $0,91^{\text {ns }}$ \\
\hline$T \times C$ & 1 & $1,41^{\mathrm{ns}}$ & $0,85^{\text {ns }}$ & $0,76^{\mathrm{ns}}$ & $9,62^{\text {ns }}$ & $0,36^{\text {ns }}$ & $0,43^{\text {ns }}$ & $2,53^{\text {ns }}$ \\
\hline Erro & 38 & 9,63 & 8,24 & 0,66 & 931,71 & 4,50 & 4,23 & 15,79 \\
\hline CV (\%) & & 36,88 & 37,93 & 10,17 & 26,71 & 14,28 & 14,82 & 11,12 \\
\hline Média & & 8,42 & 7,57 & 64,93 & 114,30 & 14,86 & 13,88 & 35,71 \\
\hline \multirow{2}{*}{ FV } & \multirow{2}{*}{ GL } & \multicolumn{7}{|c|}{ QM } \\
\hline & & DFP & NPE & $\mathrm{NF}^{2}$ & CEG & DEG & SIG & SIC \\
\hline Trat (T) & 1 & $33,00^{\text {ns }}$ & $0,05^{\text {ns }}$ & $1,04^{\text {ns }}$ & $107,46^{\text {ns }}$ & $20,51^{\text {ns }}$ & $0,00^{\text {ns }}$ & $0,01^{\text {ns }}$ \\
\hline Ciclo (C) & 1 & $7,57^{\mathrm{ns}}$ & $0,02^{\text {ns }}$ & $0,09^{\text {ns }}$ & $137,21^{\mathrm{ns}}$ & $394,90 * *$ & $0,00^{\text {ns }}$ & $0,40^{\text {ns }}$ \\
\hline$T \times C$ & 1 & $35,19^{\text {ns }}$ & $0,02^{\text {ns }}$ & $0,17^{\mathrm{ns}}$ & $109,02^{\text {ns }}$ & $0,24^{\text {ns }}$ & $0,00^{\text {ns }}$ & $0,19^{\text {ns }}$ \\
\hline Erro & 38 & 36,30 & 0,03 & 0,53 & 110,26 & 47,11 & 0,01 & 0,14 \\
\hline CV (\%) & & 17,49 & 7,45 & 40,97 & 30,93 & 14,78 & 11,32 & 42,16 \\
\hline Média & & 34,45 & 5,33 & 3,67 & 33,95 & 46,44 & 0,02 & 0,43 \\
\hline
\end{tabular}

NPF: número de dias do plantio a floraçăo; NPC: número de dias do plantio a colheita; NFC: número de dias da floraçăo a colheita; DIA: diâmetro do pseudocaule (cm); ALP: altura de planta (m); NFO: número de folhas na floraçāo; NFI: número de filhos; MCA: peso de cacho (kg); MPE: peso de pencas (kg); NFR: número de frutos; MMF: massa média do fruto (g); CFS: comprimento do fruto da segunda penca (cm); CFP: comprimento do fruto da penúltima penca $(\mathrm{cm})$; DFS: diâmetro do fruto da segunda penca $(\mathrm{mm})$; DFP: diâmetro do fruto da penúltima penca (mm); NPE: número de pencas; NFV: número de folhas vivas na colheita; CEG: comprimento do engaço $(\mathrm{cm})$; DEG: diâmetro do engaço (cm); SIG: presença de Sigatoka-amarela na floraçăo; SIC: presença de Sigatoka-amarela na colheita. ns: Não significativo. * Significativo a 5\% de probabilidade. ${ }^{* *}$ Significativo a $1 \%$ de probabilidade. $\left(^{2}\right)$ transformado para raiz $(\mathrm{x}) .\left(^{3}\right)$ transformado para raiz $(\mathrm{x}+0,5)$.

Tabela 5. Médias para as variáveis de crescimento e produção das cultivares Pacovan e Preciosa, provenientes de plantas selecionadas e irradiadas, no primeiro e segundo ciclos de produção

\begin{tabular}{|c|c|c|c|c|}
\hline \multirow{2}{*}{ Variáveis } & \multicolumn{2}{|c|}{ Pacovan } & \multicolumn{2}{|c|}{ Preciosa } \\
\hline & Selecionadas & Testemunhas & Selecionadas & Testemunhas \\
\hline NPF & $406,13 a$ & $455,85 \mathrm{a}$ & 459,25 b & $505,45 a$ \\
\hline NPC & $536,63 \mathrm{a}$ & $583,21 \mathrm{a}$ & 591,88 b & $646,64 \mathrm{a}$ \\
\hline NFC & $130,50 \mathrm{a}$ & $134,28 \mathrm{a}$ & $140,25 \mathrm{a}$ & $141,73 \mathrm{a}$ \\
\hline DIA & $15,88 b$ & $18,31 \mathrm{a}$ & $18,34 \mathrm{a}$ & $19,77 \mathrm{a}$ \\
\hline ALP & $2,57 \mathrm{a}$ & $3,12 b$ & $2,64 \mathrm{a}$ & $3,07 \mathrm{~b}$ \\
\hline NFO & $10,00 \mathrm{a}$ & $9,43 a$ & $9,50 \mathrm{a}$ & $11,00 \mathrm{a}$ \\
\hline NFI & $3,13 \mathrm{a}$ & $2,78 \mathrm{a}$ & $2,75 \mathrm{a}$ & $2,55 \mathrm{a}$ \\
\hline MCA & $6,74 a$ & $7,38 \mathrm{a}$ & $6,90 \mathrm{a}$ & $8,91 \mathrm{a}$ \\
\hline MPE & $6,03 \mathrm{a}$ & $6,44 \mathrm{a}$ & $6,08 \mathrm{a}$ & $8,04 \mathrm{a}$ \\
\hline NFR & $56,50 \mathrm{~b}$ & $68,56 a$ & $56,50 \mathrm{~b}$ & $67,73 \mathrm{a}$ \\
\hline MMF & $108,57 \mathrm{a}$ & $95,65 \mathrm{a}$ & $105,06 \mathrm{a}$ & $117,20 \mathrm{a}$ \\
\hline CFS & $13,75 \mathrm{a}$ & $14,04 \mathrm{a}$ & $15,00 \mathrm{a}$ & $14,85 \mathrm{a}$ \\
\hline CFP & $12,63 a$ & $12,89 a$ & $13,88 a$ & $13,88 a$ \\
\hline DFS & $35,00 \mathrm{a}$ & $33,21 \mathrm{a}$ & $33,38 \mathrm{a}$ & $36,36 \mathrm{a}$ \\
\hline DFP & $34,38 \mathrm{a}$ & $32,13 a$ & $32,63 a$ & $34,97 \mathrm{a}$ \\
\hline NPE & $5,25 \mathrm{a}$ & $5,67 \mathrm{a}$ & $5,00 \mathrm{a}$ & $5,45 \mathrm{a}$ \\
\hline NFV & $3,50 \mathrm{a}$ & $3,24 a$ & $4,63 a$ & $3,55 a$ \\
\hline CEG & $34,88 \mathrm{a}$ & $37,61 \mathrm{a}$ & $37,25 a$ & $33,06 \mathrm{a}$ \\
\hline DEG & $41,63 b$ & $48,07 \mathrm{a}$ & $45,00 \mathrm{a}$ & $47,02 \mathrm{a}$ \\
\hline SIG & $1,00 \mathrm{~b}$ & $0,32 \mathrm{a}$ & $0,00 \mathrm{a}$ & $0,00 \mathrm{a}$ \\
\hline SIC & $1,25 \mathrm{a}$ & $1,07 \mathrm{a}$ & $0,50 a$ & $0,42 \mathrm{a}$ \\
\hline
\end{tabular}

NPF: número de dias do plantio a floração; NPC: número de dias do plantio a colheita; NFC: número de dias da floração a colheita; DIA: diâmetro do pseudocaule (cm); ALP: altura de planta (m); NFO: número de folhas na floração; NFI: número de filhos; MCA: massa de cacho (kg); MPE: massa de pencas (kg); NFR: número de frutos; MMF: massa média do fruto (g); CFS: comprimento do fruto da segunda penca (cm); CFP: comprimento do fruto da penúltima penca (cm); DFS: diâmetro do fruto da segunda penca $(\mathrm{mm})$; DFP: diâmetro do fruto da penúltima penca $(\mathrm{mm})$; NPE: número de pencas; NFV: número de folhas vivas na colheita; CEG: comprimento do engaço $(\mathrm{cm})$; DEG: diâmetro do engaço $(\mathrm{cm})$; SIG: presença de Sigatoka-amarela na floraçáo; SIC: presença de Sigatoka-amarela na colheita. ns: Não significativo. * Significativo a 5\% de probabilidade. ${ }^{* *}$ Significativo a $1 \%$ de probabilidade. Transformado para raiz $(\mathrm{x})$. Transformado para raiz $(\mathrm{x}+0,5)$. 


\section{CONCLUSÃO}

Pelos resultados, verifica-se em algumas plantas irradiadas porte reduzido e outras características agronômicas semelhantes às testemunhas não irradiadas, indicando ser possível selecionar plantas mutantes com características agronômicas superiores e de porte baixo, tanto para 'Pacovan' quanto para 'Preciosa'.

\section{REFERÊNCIAS}

BERMÚDEZ, I.; HERRERA, I.; ORELLANA, N.; VEITIA, N.; ROMERO, C.; CLAVELO, J.; GARCIA, L.; ACOSTA, M.; GARCIA, L.R.; PADRÓN, Y. Study of experimentally-induced variants of 'Manzano' (AAB) and 'Gros Michel' (AAA) bananas for their potencial resistance to Fusarium wilt. Infomusa, v.11, p.7-8, 2002.

DONATO, S.L.R.; SILVA, S.O.; LUCCA FILHO, O.A.; LIMA, M.B.; DOMINGUES, H.; ALVES, J.S. Comportamento de variedades e híbridos de bananeira (Musa spp.), em dois ciclos de produção no sudoeste da Bahia. Revista Brasileira de Fruticultura, v.28, p.139-144, 2006.

FAO/IAEA. Mutant Varieties databases. Disponível em: <http:// www.mvd.iaea.org/MVD.htm>. Acesso em: 6/4/2011.

FAO. Food and agriculture organization of the United Nations. Disponível em: <faostat.fao.org/site/567/DesktopDefault. aspx?PageID=567>. Acesso em: 6/4/2011.

GAUHL, F.; PASBERG-GAUHL, C.; VUYLSTEKE, D.; ORTIZ, $\mathrm{R}$. Multiplicational evaluation of black sigatoka resistance in banana and plantain. Abuja: International Institute of Tropical Agriculture. 1993. 59p. (Research Guide 47)
HO, Y.M.; MAK, C.; LIEW, K.W. Selection of banana cultivars for tolerance to fusarium wilt race 4 in Malaysia. In: MOLINA, A. B.; NIK MASDEK, N. H.; LIEW, K. W. (Ed.). Banana Fusarium wilt management: towards sustainable cultivation. Los Baños, Laguna: International Network for the Improvement of Bananas and Plantains - Asia and Pacific Network, 2001. p.234-238.

LIMA, M.B.; SILVA, S.O.; JESUS, O.N.; OLIVEIRA, W.S.J.; GARRIDO, M.S.; AZEVEDO, R.L. Avaliação de cultivares e híbridos de bananeira no recôncavo baiano. Ciência e Agrotecnologia, v.29, p.515-520, 2005.

PESTANA, R.K.N.; AMORIM, E.P.; SILVA, S.O.; TULMANN NETO, A. Irradiação gama para mutagênese in vitro em bananeira 'Terra Maranhão'. Pesquisa Agropecuária Brasileira, v.45, p.13281330, 2010.

SAS LEARNING EDITION Programa SAS - Getting started with the SAS Learning Edition. North Carolina: Cary SAS Publishing, 2002. 200p.

SILVA, S.O.; FLORES, J.C.O.; Lima Neto, F.P. Avaliaçăo de cultivares e híbridos de bananeira em quatro ciclos de produção. Pesquisa Agropecuária Brasileira, v.37, p. 1567-1574, 2002.

SILVA, S.O.; GASPAROTTO, L.; MATOS, A.P.; CORDEIRO, Z.J.M.; FERREIRA, C. F.; RAMOS, M.M.; JESUS, O.N. Programa de Melhoramento de Bananeira no Brasil - Resultados Recentes. Cruz das Almas: Embrapa Mandioca e Fruticultura, 2003. 36p. (Documentos 123)

STOVER, R. H. Banana, plantain, and abaca diseases. Kew, Surrey, England: Commonwhealth Mycology Institute, 1972. 316p.

TANG, C.Y.; TAI, C.H. Improvement of the horticultural traits of Cavendish banana (Musa spp, AAA group) through somaclonal variation. Tropical Agriculture, v.78, p.40-47, 2001. 